Bull. Austral. Math. Soc.

$30 \mathrm{~F} 40,20 \mathrm{H} 10$

VoL. $71(2005) \quad[29-35]$

\title{
TWO NECESSARY AND SUFFICIENT CONDITIONS FOR THE EXTENSION OF MÖBIUS GROUPS
}

\author{
Xiantao Wang and Shouyao Xiong
}

Let $\operatorname{SL}\left(2, \Gamma_{n}\right)$ be the $n$-dimensional Clifford matrix group and $G \subset \operatorname{SL}\left(2, \Gamma_{n}\right)$ be a non-elementary subgroup. We show that $G$ is the extension of a subgroup of $\operatorname{SL}(2, \mathbb{C})$ if and only if $G$ is conjugate in $\operatorname{SL}\left(2, \Gamma_{n}\right)$ to a group $G^{\prime}$ which satisfies the following properties:

(1) there exist loxodromic elements $g_{0}, h \in G^{\prime}$ such that fix $\left(g_{0}\right)=\{0, \infty\}$, $\operatorname{fix}\left(g_{0}\right) \cap \operatorname{fix}(h)=\emptyset$ and $\operatorname{fix}(h) \cap \mathbb{C} \neq \emptyset$;

(2) $\operatorname{tr}(g) \in \mathbb{C}$ for each loxodromic element $g \in G^{\prime}$.

Further $G$ is the extension of a subgroup of $\operatorname{SL}(2, \mathbb{R})$ if and only if $G$ is conjugate in $\mathrm{SL}\left(2, \Gamma_{n}\right)$ to a group $G^{\prime}$ which satisfies the following properties:

(1) there exists a loxodromic element $g_{0} \in G^{\prime}$ such that $\operatorname{fix}\left(g_{0}\right) \cap\{0, \infty\}$ $\neq \emptyset$

(2) $\operatorname{tr}(g) \in \mathbb{R}$ for each loxodromic element $g \in G^{\prime}$.

The discreteness of subgroups of $\operatorname{SL}\left(2, \Gamma_{n}\right)$ is also discussed.

\section{INTRODUCTION AND MAIN RESULTS}

As in [1] or [8], let $\mathrm{SL}\left(2, \Gamma_{n}\right)$ denote the $n$-dimensional Clifford matrix group and $M\left(\bar{R}^{n}\right)$ the full group of $n$-dimensional sense-preserving Möbius transformations.

In the study of higher dimensional Möbius groups, the following two problems are fundamental and interesting.

PROBLEM 1. When is a subgroup $G \subset \mathrm{SL}\left(2, \Gamma_{n}\right)$ the extension of a group of $\mathrm{SL}(2, \mathbb{R})$ ?

Problem 2. When is a subgroup $G \subset \operatorname{SL}\left(2, \Gamma_{n}\right)$ the extension of a group of $\operatorname{SL}(2, \mathbb{C})$ ?

Here $G$ is called the extension of a subgroup of $\operatorname{SL}(2, \mathbb{C})$ (or $\operatorname{SL}(2, \mathbb{R})$ ) if $G$ is conjugate in $\mathrm{SL}\left(2, \Gamma_{n}\right)$ to a subgroup of $\mathrm{SL}(2, \mathbb{C})$ (or $\mathrm{SL}(2, \mathbb{R})$, respectively).

Many authors have discussed these two problems. For Problem 1, when $n=2$, Maskit ([6]) proved

Received 19th July, 2004

The research was partly supported by the N.S. Foundations of China (No. 10271043) and Zhejiang province.

Copyright Clearance Centre, Inc. Serial-fee code: 0004-9727/05 \$A2.00+0.00. 
TheOREM M. Let $G \subset \mathrm{SL}(2, \mathbb{C})$ be a Kleinian group in which $\operatorname{tr}^{2}(g) \geqslant 0$ for all $g \in G$. Then $G$ is Fuchsian.

When $n \geqslant 2$, Apanasov ([2]) proved

THEOREM A. If $G \subset \mathrm{SL}\left(2, \Gamma_{n}\right)$ is non-elementary and each nontrivial element of $G$ is either hyperbolic or strictly parabolic or strictly elliptic, then $G$ is the extension of a group of $\mathrm{SL}(2, \mathbb{R})$.

Subsequently, we generalised Theorems $M$ and $A$ into the following form, (see [7]).

ThEOREM WY. Let $G \subset \operatorname{SL}\left(2, \Gamma_{n}\right)$ be non-elementary. If each loxodromic element of $G$ is hyperbolic, then $G$ is the extension of a group of $\operatorname{SL}(2, \mathbb{R})$.

It is well-known that the trace of an element of $\mathrm{SL}(2, C)$ is conjugate invariant in $\mathrm{SL}(2, C)$. This property does not hold in $\mathrm{SL}\left(2, \Gamma_{n}\right)$ when $n \geqslant 3$. In order to overcome this difficulty, Theorems $A$ and $W Y$ require that each loxodromic element of $G$ is hyperbolic, since the trace of a hyperbolic element is conjugate invariant in $\operatorname{SL}\left(2, \Gamma_{n}\right)$. A natural problem is how to characterise subgroups of $\operatorname{SL}\left(2, \Gamma_{n}\right)$ without requiring each loxodromic element be hyperbolic. As the first aim of this paper, we shall consider this problem. By using different method, we shall prove

THEOREM 1. Let $G \subset \mathrm{SL}\left(2, \Gamma_{n}\right)$ be non-elementary. Then $G$ is the extension of a group of $\operatorname{SL}(2, \mathbb{R})$ if and only if $G$ is conjugate in $\operatorname{SL}\left(2, \Gamma_{n}\right)$ to $G^{\prime}$ which satisfies the following properties:

(1) there exists a loxodromic element $g_{0} \in G^{\prime}$ such that $\operatorname{fix}\left(g_{0}\right) \cap\{0, \infty\} \neq \emptyset$; and

(2) $\operatorname{tr}(g) \in \mathbb{R}$ for each loxodromic element $g \in G^{\prime}$.

COROLLARY 1. Let $G \subset \mathrm{SL}\left(2, \Gamma_{n}\right)$ be non-elementary. If each loxodromic element of $G$ is hyperbolic and each elliptic element of $G$ (if any) is of finite order, then $G$ is discrete.

REMARK 1. Obviously, Theorem 1 is a generalisation of Theorems M, A and WY. Example 1 shows the difference between Theorem 1 and Theorem WY.

Concerning Problem 2, recently Chen ([4]) proved

THEOREM C. Let $G \subset \mathrm{SL}\left(2, \Gamma_{n}\right)$ be non-elementary. If $G$ contains hyperbolic elements, then $G$ is the extension of a group of $\mathrm{SL}(2, \mathbb{C})$ if and only if $G$ is conjugate in $\mathrm{SL}\left(2, \Gamma_{n}\right)$ to $G^{\prime}$ which satisfies the following properties:

(1) there exist hyperbolic elements $g_{0}, h \in G^{\prime}$ such that fix $\left(g_{0}\right)=\{0, \infty\}$, fix $\left(g_{0}\right) \cap \operatorname{fix}(h)=\emptyset$ and fix $(h) \cap \mathbb{C} \neq \emptyset$; and

(2) $\operatorname{tr}(g) \in \mathbb{C}$ for each $g \in G^{\prime}$.

The following statement is obvious.

FACT. Each non-elementary subgroup of $\operatorname{SL}(2, \mathbb{C})$ (that is, $\operatorname{SL}\left(2, \Gamma_{2}\right)$ ) is the extension of a group of $\mathrm{SL}(2, \mathbb{C})$. 
But when $n=2$, Theorem $\mathrm{C}$ does not coincide with the above stated fact. This means that the condition " $G$ containing hyperbolic elements" in Theorem $\mathrm{C}$ is too strict. We can see from [4] that this condition plays a key role in the proof. As the second aim of this paper, we shall study Theorem $\mathrm{C}$ further and prove the following.

ThEOREM 2. Let $G \subset \operatorname{SL}\left(2, \Gamma_{n}\right)$ be non-elementary. Then $G$ is the extension of a group of $\mathrm{SL}(2, \mathbb{C})$ if and only if $G$ is conjugate in $\operatorname{SL}\left(2, \Gamma_{n}\right)$ to $G^{\prime}$ which satisfies the following properties:

(i) there exist loxodromic elements $g_{0}, h \in G^{\prime}$ such that fix $\left(g_{0}\right)=\{0, \infty\}$, fix $\left(g_{0}\right) \cap \operatorname{fix}(h)=\emptyset$ and fix $(h) \cap \mathbb{C} \neq \emptyset$; and

(ii) $\operatorname{tr}(g) \in \mathbb{C}$ for each loxodromic element $g \in G^{\prime}$.

COROLlaRY 2. Let $G \subset \operatorname{SL}\left(2, \Gamma_{n}\right)$ be non-elementary. If $G$ is conjugate in $\mathrm{SL}\left(2, \Gamma_{n}\right)$ to $G^{\prime}$ which satisfying properties (i) and (ii) as in Theorem 2 , then $G$ is discrete if and only if each non-elementary subgroup of $G$ generated by two loxodromic elements is discrete.

REMARK 2. Obviously, Theorem 2 is a generalisation of Theorem C. Also when $n=2$, Theorem 2 completely coincides with the above stated fact, since the traces of elements of $\operatorname{SL}(2, \mathbb{C})$ are invariant under the conjugation in $\mathrm{SL}(2, \mathbb{C})$.

We shall prove Theorems 1, 2 and Corollaries 1, 2 in Section 3. In Section 2, we shall introduce some necessary material which is needed in Section 3.

\section{Preliminaries}

We need the following preliminaries, see $[\mathbf{1}, \mathbf{8}]$ for more detail.

Let $\Gamma_{n}$ denote the $n$-dimensional Clifford group, $\mathrm{SL}\left(2, \Gamma_{n}\right)$ the group of all $n$ dimensional Clifford matrices and

$$
\operatorname{PSL}\left(2, \Gamma_{n}\right)=\operatorname{SL}\left(2, \Gamma_{n}\right) /\{ \pm I\}
$$

where $I$ is the unit matrix.

Let $A=\left(\begin{array}{ll}a & b \\ c & d\end{array}\right) \in \operatorname{PSL}\left(2, \Gamma_{n}\right)$ correspond to the mapping in $\bar{R}^{n}$

$$
x \mapsto A x=(a x+b)(c x+d)^{-1} .
$$

Then this is an isomorphism between $\operatorname{PSL}\left(2, \Gamma_{n}\right)$ and $M\left(\vec{R}^{n}\right)$. We shall identify the element in $M\left(\bar{R}^{n}\right)$ with its corresponding element in $\operatorname{PSL}\left(2, \Gamma_{n}\right)$.

In the following, we shall consider a more general case; that is, we shall consider subgroups in $\operatorname{SL}\left(2, \Gamma_{n}\right)$ instead of those in $\operatorname{PSL}\left(2, \Gamma_{n}\right)$. 
A nontrivial element $f=\left(\begin{array}{ll}a & b \\ c & d\end{array}\right) \in \mathrm{SL}\left(2, \Gamma_{n}\right)$ is called is loxodromic if $f$ is conjugate in $\operatorname{SL}\left(2, \Gamma_{n}\right)$ to $\left(\begin{array}{cc}r \lambda & 0 \\ 0 & r^{-1} \lambda^{\prime}\end{array}\right)$, where $r>0, r \neq 1, \lambda \in \Gamma_{n}$ and $|\lambda|=1$; in particular, we say that $f$ is hyperbolic if $\lambda= \pm 1$.

Let

$$
\operatorname{tr}(f)=a+d^{*} \text { and } \operatorname{fix}(f)=\left\{x \in \overline{\mathbb{R}}^{n}: f(x)=x\right\} .
$$

We say that $f$ is vectorial if $b, c \in \overline{\mathbb{R}}^{n}$. Then we have (see [1])

LEMMA 1. A nontrivial element $f$ is hyperbolic if and only if $f$ is vectorial and $\operatorname{tr}^{2}(f)>4$.

Corollary 3. Let $f=\left(\begin{array}{ll}a & b \\ c & d\end{array}\right) \in \mathrm{SL}\left(2, \Gamma_{n}\right)$ be loxodromic. Then $f$ is hyperbolic if and only if $b^{*}=b, c^{*}=c$ and $\operatorname{tr}(f) \in \mathbb{R}$.

For any loxodromic element $g \in \mathrm{SL}(2, \mathbb{C}), g$ is hyperbolic if and only if $\operatorname{tr}(g) \in \mathbb{R}$. But the following example shows that when $n>2$, this statement is not true.

EXAMPLE 1. Let

$$
g=\left(\begin{array}{cc}
2 e_{1} e_{2} & 3 e_{1} e_{2} \\
e_{1} e_{2} & 2 e_{1} e_{2}
\end{array}\right)
$$

Then $g$ is loxodromic and $\operatorname{tr}(g) \in \mathbb{R}$, but $g$ is not hyperbolic.

Let $\mathbb{H}^{n+1}=\left\{x: x=x_{0}+x_{1} e_{1}+\cdots+x_{n} e_{n} \in \overline{\mathbb{R}}^{n+1}, x_{n}>0\right\}$ and $\overline{\mathbb{H}}^{n+1}=\mathbb{H}^{n+1} \cup \overline{\mathbb{R}}^{n}$.

As in [3] we call, a subgroup $G \subset \operatorname{SL}\left(2, \Gamma_{n}\right)$, elementary if there exists some $x \in \overline{\mathbb{H}}^{n+1}$ such that the $G$-orbit $G(x)=\{g(x): g \in G\}$ at $x$ is finite. Otherwise $G$ is called nonelementary. It follows from $[3,7]$ that if $G$ is non-elementary, then $G$ contains infinitely many loxodromic elements, no two of which have a common fixed points.

\section{Proofs of the main RESUlts}

Firstly, we introduce a lemma.

Lemma 2. Let $G \subset \mathrm{SL}\left(2, \Gamma_{n}\right)$ be non-elementary and $g_{0} \in G$ be loxodromic with fix $\left(g_{0}\right)=\{0, \infty\}$. If $\operatorname{tr}(g) \in \mathbb{C}$ for any loxodromic element $g \in G$, then for any $f=\left(\begin{array}{ll}a & b \\ c & d\end{array}\right) \in G, a, d \in \mathbb{C}$.

PRoOF: If $f$ interchanges the two fixed points of $g_{0}$ or $\operatorname{fix}(f) \cap \operatorname{fix}\left(g_{0}\right) \neq \emptyset$, then the result is obvious. Now we assume that $g$ does not interchange 0 and $\infty$, and $\operatorname{fix}(g) \cap\{0, \infty\}=\emptyset$. Then $\max \{|a|,|d|\}>0$ and $b c \neq 0$. To replace $f$ by $f^{-1}$ if needed, we may assume that $a \neq 0$. Then by [7, Lemma 3.3], we see that $g_{0}^{m} f$ are loxodromic for all large enough $m$. This completes the proof. 
Proof of ThEOREM 1: The proof follows from [7, Theorem 4.1] and the following lemma.

LEMma 3. Let $G^{\prime} \subset \operatorname{SL}\left(2, \Gamma_{n}\right)$ be non-elementary. If $G^{\prime}$ satisfies the following properties:

(1) there exists a loxodromic element $g_{0} \in G^{\prime}$ such that fix $\left(g_{0}\right) \cap\{0, \infty\} \neq \emptyset$;

(2) $\operatorname{tr}(g) \in \mathbb{R}$ for each loxodromic element $g \in G^{\prime}$, then each loxodromic element in $G^{\prime}$ is hyperbolic.

Proof: Without loss of generality, we may assume that

$$
g_{0}=\left(\begin{array}{cc}
r & t \\
0 & r^{-1}
\end{array}\right),
$$

where $r \in \mathbb{R},|r|>1$ and $t \in \mathbb{R}^{n}$.

By the similar reasoning as in the proof of [7, Theorem 4.1], we may assume further that $t \in \mathbb{R}$.

Let

$$
g=\left(\begin{array}{ll}
a & b \\
c & d
\end{array}\right)
$$

be any loxodromic element in $G^{\prime}$.

If $c=0$, then $g_{0}^{m} g$ are loxodromic for all large enough $m$. Condition (2) in Lemma 3 implies that $a, d \in \mathbb{R}$ and $b, c \in \overline{\mathbb{R}}^{n}$. By Corollary 3, we know that $g$ is hyperbolic.

Now we assume that $c \neq 0$. To replace $g$ by $g^{-1}$ if needed, we may assume that $g(\infty) \notin$ fix $\left(g_{0}\right)$. Then $g_{0}^{m} g$ and $g g_{0}^{m}$ are loxodromic for all sufficiently large $m$. Condition (2) in Lemma 3 implies that

$$
\left\{a+\frac{t}{r-r^{-1}} c, a+\frac{t}{r-r^{-1}} c^{*}\right\} \subset \mathbb{R} .
$$

Hence $c^{*}=c$. It follows from $\Delta(g)=a d^{*}-b c^{*}=1$ that $b^{*}=b$. Then Corollary 3 tells us that $g$ is hyperbolic.

The proof of our lemma is completed.

Proof of TheOREM 2: The necessity is obvious. In the following we prove the sufficiency.

By conditions (i) and (ii), we may assume that $g_{0}$ has the form:

$$
g_{0}=\left(\begin{array}{cc}
r & 0 \\
0 & r^{-1}
\end{array}\right),
$$

where $r \in \mathbb{C}$ and $|r|>1$.

In the following, we shall prove that $h \in \mathrm{SL}(2, C)$. 
Let

$$
h=\left(\begin{array}{ll}
a & b \\
c & d
\end{array}\right) \text {. }
$$

Then $b c \neq 0, \max \{|a|,|d|\}>0$ and $a+d^{*} \in \mathbb{C}$. Without loss of generality, we may assume that $a \neq 0$. Otherwise we replace $h$ by $h^{-1}$. Then Lemma 2 implies that $a, d \in \mathbb{C}$.

It follows from $a b^{*}$ and $a^{*} c \in \overline{\mathbb{R}}^{n}$ that $h$ has the form:

$$
h=\left(\begin{array}{cc}
a & a s \\
a^{\prime} q & d
\end{array}\right)
$$

where $s=s_{0}+\sum_{i=2}^{n-1} s_{i} e_{i}\left(s_{0} \in \mathbb{C}, s_{i} \in \mathbb{R}\right), q=q_{0}+\sum_{i=2}^{n-1} q_{i} e_{i}\left(q_{0} \in \mathbb{C}, q_{i} \in \mathbb{R}\right)$.

Now $\Delta(h)=a d^{*}-(a s)\left(a^{\prime} q\right)^{*}=1$ implies that $s q \in \mathbb{C}$. Hence $s \in \mathbb{C}$ if and only if $q \in \mathbb{C}$, since $s q \neq 0$.

It follows from das $\in \overline{\mathbb{R}}^{n}$ that $a d \in \mathbb{R}$ or $s \in \mathbb{C}$. We claim that $s \in \mathbb{C}$. Suppose $s \notin \mathbb{C}$. Then $a d \in \mathbb{R}$. We may assume that

$$
d=k a^{\prime}
$$

where $k \in \mathbb{R}$. Then we have

$$
h=\left(\begin{array}{cc}
a & a s \\
a^{\prime} q & k a^{\prime}
\end{array}\right) .
$$

This implies that $s q \in \mathbb{R}$. Hence there exists $k_{1} \in \mathbb{R}$ such that $q=k_{1} s^{\prime}$. Under the conjugation of a suitable element in $\operatorname{SL}(2, \mathbb{R})$, we may assume that

$$
g=\left(\begin{array}{cc}
r & 0 \\
0 & r^{-1}
\end{array}\right) \text { and } h=\left(\begin{array}{cc}
a & a s \\
\varepsilon a^{\prime} s^{\prime} & k a^{\prime}
\end{array}\right)
$$

where $r \in \mathbb{C},|r|>1, \varepsilon= \pm 1, s=s_{0}+\sum_{i=2}^{n-1} s_{i} e_{i}, s_{0} \in \mathbb{C}$ and $s_{i}, k \in \mathbb{R}$.

We see from fix $(h) \cap \mathbb{C} \neq \emptyset$ and $h$ being loxodromic that $s_{0} \neq 0$ and $a^{\prime}=-\varepsilon a$. Hence

$$
h=\left(\begin{array}{cc}
a & a s \\
-a s^{\prime} & -\varepsilon k a
\end{array}\right) .
$$

Since $h^{2}$ is loxodromic, by Lemma 2 , we know that $a^{2}-a s a s^{\prime} \in \mathbb{C}$, which implies that sas' $\in \mathbb{C}$. Then $\bar{a}=a$, that is, $a \in \mathbb{R} \backslash\{0\}$. By Corollary $3, h$ is hyperbolic. Then, by [1],

$$
\operatorname{fix}(h)=\left\{t_{1} s, t_{2} s\right\}
$$

where $t_{1,2}=-\left[(1+\varepsilon k) a \pm \sqrt{(1-k \varepsilon)^{2} a^{2}-4}\right] / 2 a^{-1}|s|^{-2} \in \mathbb{R}$.

Condition $(i)$ implies that $s \in \mathbb{C}$. This contradiction shows that $s \in \mathbb{C}$. 
Our claim implies that $h$ has the following form:

$$
h=\left(\begin{array}{ll}
a & b \\
c & d
\end{array}\right),
$$

where $a, b, c, d \in \mathbb{C}$ with $b c \neq 0$.

For any nontrivial element

$$
p=\left(\begin{array}{ll}
u & v \\
\alpha & \beta
\end{array}\right) \in G^{\prime},
$$

by Lemma 2, we know that $u, \beta \in \mathbb{C}$. By considering $p g$, Lemma 2 implies that $v, \alpha \in \mathbb{C}$. This shows that $p \in \mathrm{SL}(2, \mathbb{C})$ which completes the proof.

Proof of Corollary 1: If each loxodromic element of $G$ is hyperbolic, then Theorem 1 yields that $G$ is conjugate in $\operatorname{SL}\left(2, \Gamma_{n}\right)$ to a group $G^{\prime}$ of $\operatorname{SL}(2, \mathbb{R})$. Then $[\mathbf{5}$, Theorem 2] or [3, Theorem 8.4.1] implies that $G^{\prime}$ is discrete. Hence $G$ is discrete.

ProOF OF COROllary 2: The proof follows from [9, Theorem 2].

\section{REFERENCES}

[1] L.V. Ahlfors, 'On the fixed points of Möbius transformations in $\bar{R}^{n}$, Ann. Acad. Sci. Fenn. Ser. A I Math. 10 (1985), 15-27.

[2] B.N. Apanasov, The geometry of discrete groups and manifolds (Nauka, Moscow, 1991).

[3] A.F. Beardon, The geometry of discrete groups, Graduate text in Mathematics 91 (Springer-Verlag, Berlin, Heidelberg, New York, 1983).

[4] M. Chen, 'The extension of Möbius groups', Complex Var. Theory Appl. 47 (2002), 225-228.

[5] T. Jørgensen, 'A note on subgroups of SL(2, C)', Quart. J. Math. Oxford Ser. 228 (1977), 209-212.

[6] B. Maski, Kleinian groups, Grundlehren der Mathematischen Wissenschaften 287 (Springer-Verlag, Berlin, Heidelberg, New York, 1988).

[7] X. Wang and W. Yang,, 'Generating systems of subgroups in PSL $\left(2, \Gamma_{n}\right)$ ', Proc. Edinburgh Math. Soc. 45 (2002), 49-58.

[8] X. Wang and W. Yang, 'Discreteness Criteria of Mobius groups of high dimensions and convergence theorems of Kleinian groups', Adv. Math. 159 (2001), 68-82.

[9] X. Wang and W. Yang, 'Discrete criteria for subgroups in $S L(2, C)$ ', Math. Proc. Cambridge. Philos. Soc. 124 (1998), 51-55.

Department of Mathematics

Hunan Normal University

Changsha, Hunan 410081

People's Republic of China

e-mail: xtwang@hunnu.edu.cn
Department of Mathematics

Changsha University of Science and Technology

Changsha, Hunan 410000

People's Republic of China 